\title{
Nuances in Training During the Age of Teledermatology
}

\author{
Young H. Lim, MD, PhD
}

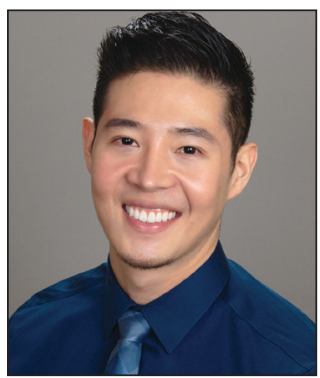

\section{RESIDENT PEARL}

- The COVID-19 pandemic has accelerated the adoption of teledermatology, enhancing patient access to dermatologic care while also facilitating multidisciplinary discourse and providing opportunities for education and training. However, these virtual interactions require a vigilance for patient privacy and security with an added emphasis on visual diagnostics to deliver high-quality care.
COVID-19 was declared a global pandemic by the World Health Organization on March 11, 2020, and within the following 2 months, most states had issued mandatory stay-at-home orders, leaving many patients without access to outpatient care. Similar to many other outpatient-based specialties, dermatology quickly adopted telemedicine into clinical practice, and this expansion of virtual communication provided (1) increased access for patients living in remote areas, (2) ease of multidisciplinary collaboration, and (3) new opportunities for training and education. This article highlights the caveats of teledermatology, such as the need for excellent visual diagnostic skills and the vulnerability of patient privacy and protected information. Additionally, as teledermatology is poised to further expand in the future, it is critical to reflect on patient safety as well as its clinical efficacy in comparison to in-person evaluation.

Cutis. 2022;109:E43-E45.

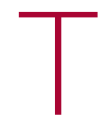

he COVID-19 pandemic largely altered the practice of medicine, including a rapid expansion of telemedicine following the March 2020 World Health Organization guidelines for social distancing, which recommended suspension of all nonurgent in-person visits. ${ }^{1}$
Expectedly, COVID-related urgent care visits initially comprised the bulk of the new telemedicine wave: NYU Langone Health (New York, New York), for example, saw a $683 \%$ increase in virtual visits between March and April 2020, most (55.3\%) of which were for respiratory concerns. In-person visits, on the other hand, concurrently fell by more than $80 \%$. Interestingly, nonurgent ambulatory care specialties also saw a considerable uptick in virtual encounters, from less than 50 visits in a typical day to an average of 7000 in a 10-day stretch. ${ }^{2}$

As a largely ambulatory specialty that relies on visual examination, dermatology was no exception to the swing toward telemedicine, or teledermatology (TD). Before the COVID-19 pandemic, 14.1\% (82 of 582 respondents) of practicing US dermatologists reported having used teledermatology, compared to $96.9 \%$ (572/591) during the pandemic. ${ }^{3}$ Even at my home institution (Massachusetts General Hospital [Boston, Massachusetts] and its 12 affiliated dermatology clinics), the number of in-person visits in April $2020(n=67)$ was less than 1\% of that in April $2019(n=7919)$, whereas there was a total of 1564 virtual visits in April 2020 compared to zero the year prior. Virtual provider-to-provider consults (e-consultations) also saw an increase of more than $20 \%$, suggesting that dermatology's avid adoption of TD also had improved the perceived accessibility of our specialty. ${ }^{4}$

The adoption and adaptation of TD are projected to continue to grow rapidly across the globe, as digitalization has enhanced access without increasing costs, shortened wait times, and even created opportunities for primary care providers based in rural or overseas locations to learn the diagnosis and treatment of skin disease. ${ }^{5}$ Residents and fellows should be privy to the nuances of training and

From the Department of Dermatology, Harvard Combined Dermatology Residency, Boston, Massachusetts.

The author reports no conflict of interest.

Correspondence: Young H. Lim, MD, PhD, 55 Fruit St, Boston, MA 02114 (ylim6@partners.org).

doi:10.12788/cutis.0461 
practicing in this digital era, as our careers inevitably will involve some facet of TD.

\section{The Art of Medicine}

Touch, a sense that perhaps ranks second to sight in dermatology, is absent in TD. In either synchronous (live-interactive, face video visits) or asynchronous (storeand-forward, where digital photographs and clinical information sent by patients or referring physicians are assessed at a later time) $\mathrm{TD}$, the skin cannot be rubbed for texture, pinched for thickness, or pushed for blanching. Instead, all we have is vision. Irwin Braverman, MD, Professor Emeritus of Dermatology at Yale University (New Haven, Connecticut), alongside Jacqueline Dolev, MD, dermatologist and Yale graduate, and Linda Friedlaender, curator at the Yale Center for British Art, founded an observational skills workshop in which trainees learn to observe and describe the paintings housed in the museum, noting all memorable details: the color of the sky, the actions of the animals, and the facial expressions of the people. A study of 90 participants over a 2-year period found that following the workshop, the ability to identify key diagnostic details from clinical photography improved by more than $10 \% .{ }^{6}$ Other studies also utilizing fine art as a medical training tool to improve "visual literacy" saw similarly increased sophistication in the description of clinical imagery, which translated to better diagnostic acumen. ${ }^{7}$ Confined to video and photographs, TD necessitates trainees and practicing dermatologists to be excellent visual diagnosticians. Although surveyed dermatologists believe TD is presently appropriate for acne, benign lesions, or follow-up appointments, ${ }^{3}$ conditions for which patients have been examined via TD have included drug eruptions, premalignant or malignant neoplasms, infections, and papulosquamous or inflammatory dermatoses. ${ }^{8}$ At the very least, clinicians should be versed in identifying those conditions that require in-person evaluation, as patients cannot be held responsible to distinguish which situations can and cannot be addressed virtually.

\section{Issues of Patient-Physician Confidentiality}

Teledermatology is not without its shortcomings; critics have noted diagnostic challenges with poor quality photographs or videos, inability to perform total-body skin examinations, and socioeconomic limitations due to broadband availability and speed. ${ }^{5,9}$ Although most of these shortcomings are outside of our control, a key challenge within the purview of the provider is the protection of patient privacy.

Much of the salient concerns regarding patientphysician confidentiality involve asynchronous TD, where store-and-forward data sharing allows physicians to download patient photographs or information onto their personal email or smartphones..$^{10}$ Although some hospital systems provide encryption software or hospitalsponsored devices to ensure security, physicians may opt to use their personal phones or laptops out of convenience or to save time. ${ }^{10,11}$ One study found that less than $30 \%$ of smartphone users choose to activate user authentication on their devices, even ones as simple as a passphrase. ${ }^{11}$ The digital exchange of information thus poses an immense risk for compromising protected health information (PHI), as personal devices can be easily lost, stolen, or hacked. Indeed, in 2015, more than 113 million individuals were affected by a breach of PHI, the majority over hacked network servers. ${ }^{12}$ With the growing diversity of mediums through which $\mathrm{PHI}$ is exchanged, such as videoconferencing and instant messaging, the potential medicolegal risks of information breach continue to climb. The US Department of Health \& Human Services urges health care providers to uphold best practices for security, including encrypting data, updating all software including antivirus software, using multifactor authentication, and following local cybersecurity regulations or recommendations. ${ }^{13}$ For synchronous TD, suggested best practices include utilizing headphones during live appointments, avoiding public wireless networks, and ensuring the provider and patient both scan the room with their device's camera before the start of the visit. ${ }^{14}$

\section{On the Horizon of Teledermatology}

What can we expect in the coming years? Increased utilization of telemedicine will translate into data that will help address questions surrounding safety, diagnostic accuracy, privacy, and accessibility. One aspect of TD in need of clarity is a guideline on payment and reimbursement, and whether TD can continue to be financially attractive to providers. Starting in 2020, the Centers for Medicare \& Medicaid Services removed geographic restrictions for reimbursement of telemedicine visits, enabling even urban-residing patients to enjoy the convenience of TD. This followed a prior relaxation of restrictions, where even prerecorded patient information became eligible for Medicare reimbursement. ${ }^{9}$ However, as virtual visits tend to be shorter with fewer diagnostic services compared to in-person visits, the reimbursement structure of TD must be nuanced, which is the subject of ongoing study and modification in the wake of the COVID-19 pandemic. $^{15}$

Another point to consider is the explosion of directto-consumer $\mathrm{TD}$, which allows patients to receive virtual dermatologic care or prescription medication without a pre-established relationship with any physician. In 2017, there were 22 direct-to-consumer TD services available to US patients in 45 states, 16 (73\%) of which provided dermatologic care for any concern while $6(27 \%)$ were limited to acne or antiaging and were largely prescription oriented. Orchestrated mostly by the for-profit private sector, direct-to-consumer companies are poorly regulated and have raised concerns over questionable practices, such as the use of non-US board-certified physicians, exorbitant fees, and failure to disclose medication side effects. ${ }^{16} \mathrm{~A}$ study of 16 direct-to-consumer telemedicine sites found substantial discordance in the suggested management of 
the same patient, and many of the services relied heavily on patient-provided self-diagnoses, such as a case where psoriasis medication was dispensed for a psoriasis patient who submitted a photograph of his syphilitic rash. ${ }^{17}$ Despite these problems, consumers show a willingness to pay out of pocket to access these services for their shorter waiting times and convenience. ${ }^{18}$ Hence, we must learn to ask about direct-to-consumer service use when obtaining a thorough history and be open to counseling our patients on the proper use and potential risks of direct-to-consumer TD.

\section{Final Thoughts}

The telemedicine industry is expected to reach more than $\$ 130$ billion by 2025 , with more than $90 \%$ of surveyed health care executives planning for the adoption and incorporation of telemedicine into their business models. ${ }^{19}$ The COVID-19 pandemic was an impetus for an exponential adoption of TD, and it would behoove current residents to realize that the practice of dermatology will continue to be increasingly digitalized within the coming years. Whether through formal training or selfassessment, we must strive to grow as proficient virtual dermatologists while upholding professionalism, patient safety, and health information privacy.

\section{REFERENCES}

1. Yeboah CB, Harvey N, Krishnan R, et al. The impact of COVID-19 on teledermatology: a review. Dermatol Clin. 2021;39:599-608.

2. Mann DM, Chen J, Chunara R, et al. COVID-19 transforms health care through telemedicine: evidence from the field. J Am Med Inform Assoc. 2020;27:1132-1135.

3. Kennedy J, Arey S, Hopkins Z, et al. Dermatologist perceptions of teledermatology implementation and future use after COVID-19: demographics, barriers, and insights. JAMA Dermatol. 2021;157:595-597.

4. Su MY, Das S. Expansion of asynchronous teledermatology during the COVID-19 pandemic. J Am Acad Dermatol. 2020;83:E471-E472.
5. Maddukuri S, Patel J, Lipoff JB. Teledermatology addressing disparities in health care access: a review [published online March 12, 2021]. Curr Dermatol Rep. doi:10.1007/s13671-021-00329-2

6. Dolev JC, Friedlaender LK, Braverman IM. Use of fine art to enhance visual diagnostic skills. JAMA. 2001;286:1020-1021.

7. Naghshineh S, Hafler JP, Miller AR, et al. Formal art observation training improves medical students' visual diagnostic skills. J Gen Intern Med. 2008;23:991-997.

8. Lee KJ, Finnane A, Soyer HP. Recent trends in teledermatology and teledermoscopy. Dermatol Pract Concept. 2018;8:214-223.

9. Wang RH, Barbieri JS, Nguyen HP, et al. Clinical effectiveness and costeffectiveness of teledermatology: where are we now, and what are the barriers to adoption? J Am Acad Dermatol. 2020;83:299-307.

10. Stevenson P, Finnane AR, Soyer HP. Teledermatology and clinical photography: safeguarding patient privacy and mitigating medico-legal risk. Med J Aust. 2016;204:198-200e1.

11. Smith KA, Zhou L, Watzlaf VJM. User authentication in smartphones for telehealth. Int J Telerehabil. 2017;9:3-12.

12. Breaches of unsecured protected health information. Health IT website. Updated July 22, 2021. Accessed January 16, 2022. https://www.healthit.gov/data/quickstats/breaches-unsecured -protected-health-information

13. Jalali MS, Landman A, Gordon WJ. Telemedicine, privacy, and information security in the age of COVID-19. J Am Med Inform Assoc. 2021;28:671-672.

14. Telehealth for behavioral health care: protecting patients' privacy. United States Department of Health and Human Services website. Updated July 2, 2021. Accessed January 16, 2022. https://telehealth .hhs.gov/providers/telehealth-for-behavioral-health/preparing -patients-for-telebehavioral-health/protecting-patients-privacy/

15. Shachar C, Engel J, Elwyn G. Implications for telehealth in a postpandemic future: regulatory and privacy issues. JAMA. 2020;323:2375-2376.

16. Fogel AL, Sarin KY. A survey of direct-to-consumer teledermatology services available to US patients: explosive growth, opportunities and controversy. J Telemed Telecare. 2017;23:19-25.

17. Resneck JS Jr, Abrouk M, Steuer M, et al. Choice, transparency, coordination, and quality among direct-to-consumer telemedicine websites and apps treating skin disease. JAMA Dermatol. 2016;152:768-775.

18. Snoswell CL, Whitty JA, Caffery LJ, et al. Consumer preference and willingness to pay for direct-to-consumer mobile teledermoscopy services in Australia [published online August 13, 2021]. Dermatology. doi:10.1159/000517257

19. Elliott T, Yopes MC. Direct-to-consumer telemedicine. J Allergy Clin Immunol Pract. 2019;7:2546-2552. 